EPJ Web of Conferences 47, 13004 (2013)

DOI: $10.1051 /$ epjconf/20134713004

(C) Owned by the authors, published by EDP Sciences, 2013

\title{
A wide planetary-mass companion to a young M3 star of the $A B$ Dor moving group
}

\author{
Marie-Eve Naud ${ }^{\mathrm{a}}$, Étienne Artigau, René Doyon, Lison Malo, Loïc Albert, \\ David Lafrenière and Jonathan Gagné
}

Département de physique and Observatoire du Mont-Mégantic, Université de Montréal, Montréal, QC H3C 3J7, Canada

\begin{abstract}
We present a planetary-mass companion found 42" (2000 AU) from a M3 star, candidate member of the young (50-120 Myr) AB Doradus moving group. It was identified through an ongoing survey with GMOS at Gemini-South, via its distinctively red $i-z$ color $(>3.51)$. The comoving status of this object was confirmed by 2 epochs of WIRCam/CFHT $J$-band images. The NIR photometry and WISE colors suggest an early-to-mid T bound companion. A NIR spectrum, taken with GNIRS at Gemini-North, confirms a mid$\mathrm{T}$ spectral type. With an estimated temperature between $900 \mathrm{~K}$ and $1200 \mathrm{~K}$, models predict a mass between 7 and $12 M_{J u p}$ for this object. The benchmark character of this planetary-mass object lies in its relatively well-constrained age and in its very wide separation, that allows in-depth studies that can help validating models and understanding similar but closer-in companions such as the ones that will be uncovered by next-generation planet finders (e.g. GPI and SPHERE).
\end{abstract}

\section{INTRODUCTION}

In the last years, planetary-mass objects were discovered in the Sun's neighborhood through direct imaging, thanks to new powerful high-contrast imaging techniques. Young stars of less than a few hundred million years, such as the ones found in young local associations, constitute ideal targets for that detection method, since the young planets that orbit them emit considerably more light than would older ones. The lowest-mass stars of these young associations are even better targets to detect planets of given masses, since the brightness ratio is then more favorable.

Recently, [1] identified many new low-mass members of young local associations ( $\beta$ Pictoris and AB Doradus moving group, as well as TW Hydrae, Tucana-Horologium, Columba, Carina and Argus associations). To do so, they used a novel Bayesian analysis that allows, for a star with given photometric ( $I$ and $J$ magnitudes) and astrometric $\left(\mu_{\alpha} \cos \delta, \mu_{\delta}\right.$, RA, DEC) characteristics, to evaluate the membership probability to an association. A confirmation of membership can be obtained if the distance and/or the radial velocity is/are measured and coincide with the the one(s) that is/are predicted by the analysis if the hypothesis is true. The reader is referred to [1] for more details.

The highly-probable low-mass members of young associations constitute the sample of the Planet Search around Young M-dwarfs (PSYM), a survey we undertook to search for planetary-mass companions. This survey comprises two observational programs, both carried at Gemini-South, in Chile. The first uses NICI [2], a high-contrast imager, and takes advantage of Angular Differential Imaging (ADI [3]) and Spectral Differential Imaging (SDI [4]) to detect companions down to $2 M_{\text {Jup }}$ between 15 and $300 \mathrm{AU}$ around the stars. Second epochs are currently being taken for this program,

\footnotetext{
ae-mail: naud@asto. umontreal.ca
} 


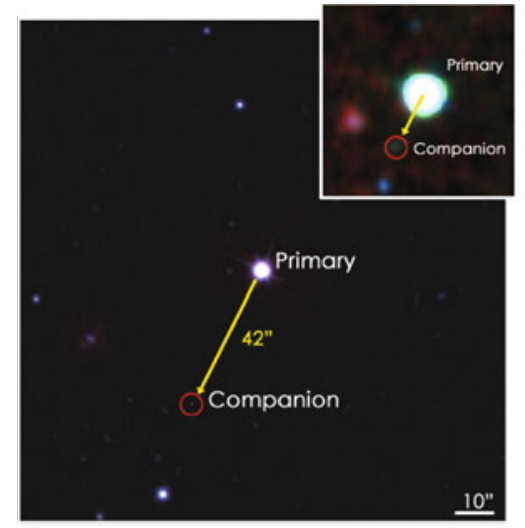

a)

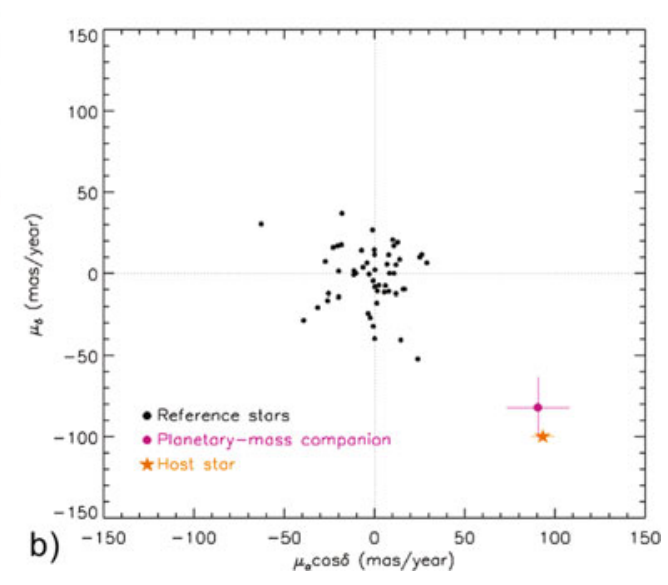

Figure 1. a) Main: the candidate in a composite image: $i$ (blue), $z$ (green) and $J$ (red). Top right corner image: a composite $W 1$ (blue), $W 2$ (green) and $W 3$ (red). As expected from a mid-T spectral type object, it is brighter in $J$ and in $W 2$. b) Proper motion as measured from two J-band images taken in October 2011 and September 2012, both with WIRCam/CFHT and with the same settings. The reference stars are represented by small black dots, while the companion is represented by a big pink dot. The host star, saturated in the $J$-band images, has literature proper motions that were averaged and shown with the orange star symbol.

Table 1. Companion photometric properties.

\begin{tabular}{|l|l||l|l||l|l|}
\hline$i_{A B}$ & $>25.28 \pm 0.07(3 \sigma)$ & $J$ & $18.12 \pm 0.03$ & $K$ & $17.36 \pm 0.03$ \\
$z_{A B}$ & $21.76 \pm 0.07$ & $H$ & $17.68 \pm 0.05$ & $W 2$ & $15.0 \pm 0.1$ \\
\hline
\end{tabular}

which surveyed over 40 stars. The second program, which allowed the discovery of the planetary-mass companion presented here, uses GMOS [5] in a standard photometric mode with $i$ and $z$ filters. It allows the identification of companions down to 3-5 $M_{J u p}$ at greater separations, between 100 and $5000 \mathrm{AU}$, through their red $i-z$ color. Over the $>90$ stars surveyed so far with GMOS, the companion presented here is the only candidate that was found.

\section{THE COMPANION AND ITS HOST STAR}

The companion was uncovered around an M3 star (Figure 1a), which was found to be a high probability candidate member of the AB Doradus moving goup (ABDMG) through the Bayesian analysis and with measurements of radial velocity. The age of ABDMG is known to be between 50 and $120 \mathrm{Myr}[6,7]$. Since the statistical distance of a star given by the analysis has proven to be a very reliable measurement of the true distance (see Fig. 5 in [1]), we adopt this value (48 $\pm 2 \mathrm{pc}$ ) for the distance of the system. The star also shows other signs that could be attributed to youth, such as very strong X-ray and $H \alpha$ emissions [8] and a very short rotation period $(\sim 1$ day, [9]).

As seen in Figure 1a, the companion is $42^{\prime \prime}$ from its host, which corresponds to an angular separation of $\sim 2000 \mathrm{AU}$ (at $48 \mathrm{pc}$ ); it is thus among the widest companions known. The candidate remained undetected in $i$ despite a deep follow-up, resulting in a very red lower limit of $>3.51$ for $i-z$. Observations in $J$ taken with WIRCam/CFHT [10] allowed the confirmation of the common proper motion between the companion and the star (Figure 1b). Table 1 summarizes the main photometric properties of the companion.

The comparison of the companion's colors to other known field dwarfs, for example $z_{A B}-J$ on Fig. 2 of [11] or $H-W 2$ to Fig. 1 of [12] indicates a spectral type between T2 and T6. A preliminary 


\section{Hot Planets and Cool Stars}

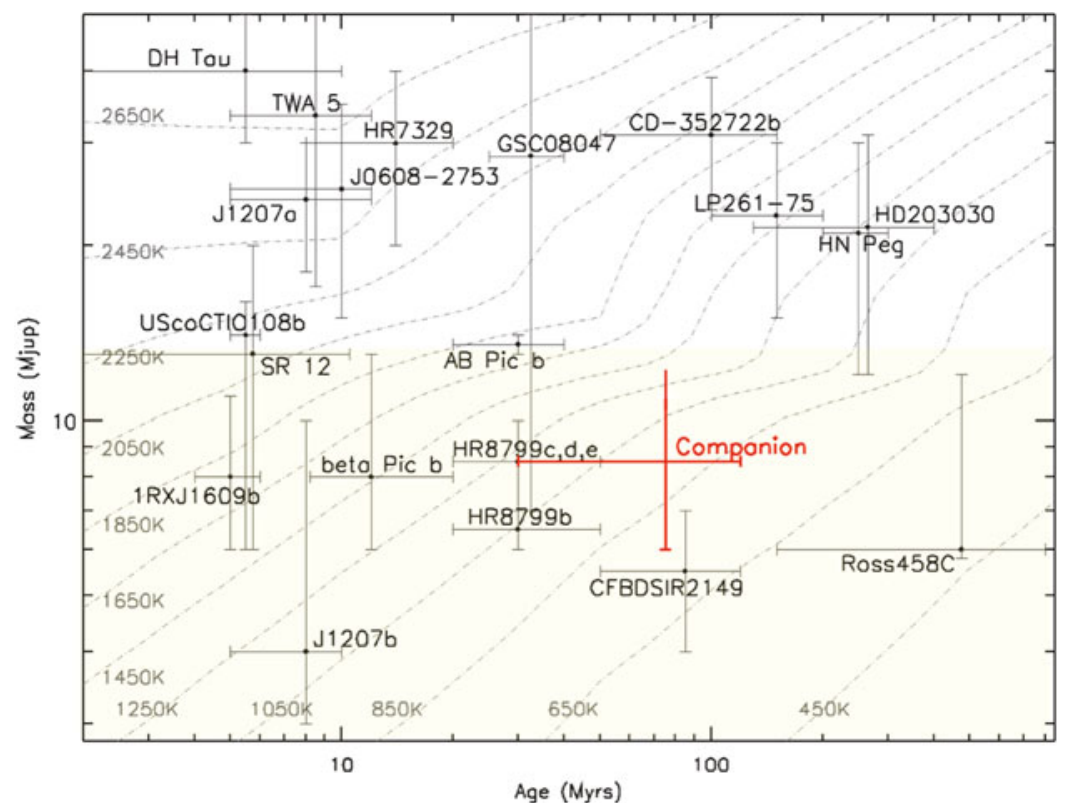

Figure 2. Mass vs age of a sample of companions and low-mass objects known along with iso- $T_{\text {eff }}$ from [15] (dashed line). Our companion is shown by a red cross. It stands in the yellow portion, which is the planetary-mass regime, according to the $13 M_{\text {Jup }}$ convention.

analysis of the near-IR (1-2.5 $\mu \mathrm{m})$ spectrum recently obtained with GNIRS/Gemini-North confirms a mid-T type. Also, colors suggest the object might have a low surface gravity (which would be consistent with a bigger radius and thus youth) and/or a high metallicity (see for example Fig. 4 in [13]). This is further suggested by the enhanced $K$-band flux observed in the GNIRS spectrum, but has still to be confirmed by a thorough analysis of the spectrum. The spectral type, or the absolute magnitudes (as computed with the statistical distance) can be combined to the age estimate of ABDMG to evaluate a mass in the 7 to $12 M_{J u p}$ range for this object (using BT-Settl [14] grids).

It is interesting to compare the physical properties of this object with that of other companions and low-mass objects (Figure 2). It is similar in some respect to Ross 458(AB) c (also called Ross 458 C), a T8.5 object that orbits a pair of M dwarfs at a distance $>1100 \mathrm{AU}[16,17]$. However, the much better constrained age of our companion could be helpful to validate models and make comparisons. Also, this object is similar, in age and mass, to the HR 8799 planets, which were among the first to be directly imaged [18], but at a very wide separation of $\sim 2000 \mathrm{AU}$, it is amenable to more detailed measurements (e.g. high-resolution spectroscopy) than these closer-in planets. It can also be seen as a more massive version of the free-floater planetary-mass object (4-7 $M_{\text {Jup }}$ ) CFBDSIR2149-0403 recently discovered in ABDMG [11], the main difference being that it is gravitationally bound. Because it is relatively bright, this object could serve as a proxy for planets such as the ones that will be uncovered by high-contrast imagers (GPI, SPHERE) and other objects that are in the glare of their host star.

\section{CONCLUSION}

We have identified a planetary-mass companion around a young $\mathrm{M}$ dwarf through a direct imaging survey. Its near-IR spectrum and other observational properties suggest that it has a mass between 7 and $12 M_{\text {Jup }}$. A thorough study of the host star, including parallax measurement and research of other 
companions, are underway. The well constrained age and wide angular separation of this new object from its host star make it a unique benchmark target to improve our understanding of exoplanets.

\section{References}

[1] L. Malo, R. Doyon, D. Lafrenière, É. Artigau, J. Gagné, F. Baron, A. Riedel, ApJ 762, 88 (2013)

[2] C. Ftaclas, E.L. Martín, D. Toomey, Proceedings of IAU Symposium \#211 211, 521 (2003)

[3] C. Marois, D. Lafrenière, R. Doyon, B. Macintosh, D. Nadeau, ApJ 641, 556 (2006)

[4] C. Marois, R. Doyon, D. Nadeau, R. Racine, M. Riopel, P. Vallee, D. Lafrenière, PASP 117, 745 (2005)

[5] I.M. Hook, I. Jørgensen, J.R. Allington Smith, R.L. Davies, N. Metcalfe, R.G. Murowinski, D. Crampton, PASP 116, 425 (2004)

[6] B. Zuckerman, I. Song, M.S. Bessell, AJ 613, L65 (2004)

[7] C.A.O. Torres, G.R. Quast, C.H.F. Melo, M.F. Sterzik, Handbook of Star Forming Regions, Volume II: The Southern Sky ASP Monograph Publications 5, 757 (2008)

[8] B. Riaz, J.E. Gizis, J. Harvin, AJ 132, 866 (2006)

[9] A.J. Norton, P.J. Wheatley, R.G. West, C.A. Haswell, R.A. Street, A. Collier Cameron, D.J. Christian, W.I. Clarkson, B. Enoch, M. Gallaway et al., A\&A 467, 785 (2007)

[10] P. Puget, E. Stadler, R. Doyon, P. Gigan, S. Thibault, G. Luppino, G. Barrick, T. Benedict, T. Forveille, W. Rambold et al., Proceedings of SPIE 5494, 978 (2004)

[11] P. Delorme, J. Gagné, L. Malo, C. Reylé, É. Artigau, L. Albert, T. Forveille, X. Delfosse, F. Allard, D. Homeier, AJ (2012)

[12] A. Mainzer, M.C. Cushing, M.F. Skrutskie, C.R. Gelino, J.D. Kirkpatrick, T. Jarrett, F. Masci, M.S. Marley, D. Saumon, E.L. Wright et al., ApJ 726, 30 (2011)

[13] S.K. Leggett, L. Albert, É. Artigau, B. Burningham, X. Delfosse, P. Delorme, T. Forveille, P.W. Lucas, M.S. Marley, D.J. Pinfield et al., AJ 448, 913 (2011)

[14] F. Allard, D. Homeier, B. Freytag, Phil. Trans. R. Soc. A 370, 2765 (2012)

[15] I. Baraffe, G. Chabrier, T.S. Barman, F. Allard, P.H. Hauschildt, A\&A 402, 701 (2003)

[16] B. Goldman, S. Marsat, T. Henning, C. Clemens, J. Greiner, MNRAS 405, 1140 (2010)

[17] R.D. Scholz, A\&A 515, A92 (2010)

[18] C. Marois, B. Macintosh, T.S. Barman, B. Zuckerman, I. Song, J. Patience, D. Lafrenière, R. Doyon, Science 322, 1348 (2008) 